\title{
FLAVONOIDS AND LIGNANS FROM LEAVES OF CRYPTOMERIA JAPONICA
}

\author{
Wen-ChiUng Su, Jim-Min FANG and YU-Shia Cheng* \\ Department of Chemistry, National Taiwan University, Taipei, Taiwan 106, Republic of China
}

(Received in revised form 27 February 1995)

Key Word Index—Cryptomeria japonica; Taxodiaceae; leaves; flavonoids; lignans.

\begin{abstract}
Eight flavonoids and 10 lignans were isolated from the leaves of Cryptomeria japonica. The new compounds are cis-dihydrodehydrodiconiferyl alcohol triacetate and secodihydrodehydrodiconiferyl alcohol tetraacetate. Their structures were determined by chemical and spectral methods.
\end{abstract}

\section{INTRODUCTION}

The Japanese cedar, Cryptomeria japonica: is a widely distributed conifer called 'sugi' in Japanese. We recently reported the isolation of sesquiterpenes [1], diterpenes of abietane-, kaurane- and labdane-types [2, 3], and a triterpene, chamaecydin [4], from the ethyl acetatesoluble part of its leaves. We describe herein 18 constituents comprised of flavonoids and lignans, including two novel compounds 16 and 17.

\section{RESULTS AND DISCUSSION}

The leaves of $C$. japonica were extracted with acetone. The ethyl acetate-soluble portion of the extract was chromatographed to give flavonoids and lignans 1-18. The known flavonoids, taxifolin (1) [5], 5-hydroxy-4',7dimethoxyflavone (2) [6], 5-hydroxy-3,4,7-trimethoxyflavone (3) [7], 5-hydroxy-3,3', $4^{\prime}, 7$-tetramethoxyflavone (4) [8], quercetin (5) [9], catechol pentaacetate (6) [10], epicatechol pentaacetate (7) [10] and $4^{\prime}, 4^{\prime \prime \prime}, 7,7^{\prime \prime}$-tetramethylamentoflavone (8) [11], the known lignans, matairesinol (9) [12], nortrachelogenin (10) [13], isolariciresinol tetraacetate (11) [14], secoisolariciresinol tetraacetate (12) [14], cedrusinin triacetate (13) [15], dihydrodehydrodiconiferyl alcohol triacetate (14) [16], cedrusin tetraacetate (15) [16] and agatharesinol tetraacetate (18) [17], were identified by comparison of their physical and spectral data (mp, $[\alpha]$, mass, IR, ${ }^{1} \mathbf{H}$ and ${ }^{13} \mathrm{CNMR}$ ) with literature data.

Compound $16\left(\mathrm{C}_{26} \mathrm{H}_{30} \mathrm{O}_{9}\right)$ was assigned as cis-dihydrodehydrodiconiferyl alcohol triacetate because it showed characteristic IR and NMR spectra (Table 1) similar to those of the trans-isomer 14. The cis-configura-

*Author to whom correspondence should be addressed. tion of 16 was established by irradiation of $\mathrm{H}-7$ (at $\delta 5.82$ ) which caused a $6.5 \%$ nOe of $\mathrm{H}-8$ (at $\delta 3.80$ ). Due to the shielding effects of the adjacent groups, the C-9 protons (at $\delta 3.82$ and 3.92) and the C-9-OAc (at $\delta 1.81$ ) of 16 appeared at higher fields than those of trans-isomer 14, occurring at $\delta 4.22,4.38$ and 2.05. The CD spectrum of 16 is similar to that of 14, exhibiting a negative Cotton effect at $276.5 \mathrm{~nm}$ and a positive Cotton effect at $253 \mathrm{~nm}$. Accordingly, compound 16 has the $(7 S, 8 S)$-configuration.

Compound $17\left(\mathrm{C}_{28} \mathrm{H}_{34} \mathrm{O}_{10}\right)$ showed IR absorptions at 1756 and $1730 \mathrm{~cm}^{-1}$ and ${ }^{13} \mathrm{C}$ signals at $\delta 168.8,168.9$, 169.1 and 171.0 attributable to four acetoxy groups. By analysis of the ${ }^{1} \mathrm{H},{ }^{13} \mathrm{C}$ and $\mathrm{H}-\mathrm{C}$ correlated spectra, the structure of 17 was determined to be secodihydrodehydrodiconiferyl alcohol tetraacetate. Compound 17 was correlated with 14 by a sequence of hydrogenolysis, giving a tetraol 17a, and acetylation (Scheme 1). This correlation confirms that $\mathbf{1 7}$ has the $8 S$-configuration. Its CD spectrum showed a negative Cotton effect at $286.5 \mathrm{~nm}$ and a positive Cotton effect at $264.5 \mathrm{~nm}$.

\section{EXPERIMENTAL}

General. Merck silica gel $60 \mathrm{~F}$ sheets were used for analytical TLC. HPLC was carried out on a Hibar Lichrosorb Si $60(7 \mu \mathrm{m}$ or $10 \mu \mathrm{m})$ column $(25 \mathrm{~cm} \times 1 \mathrm{~cm})$.

Plant material. The plant used in this study was introduced from Japan and cultivated in suburban Taipei. A voucher specimen is deposited in our laboratory. Leaves $(1.4 \mathrm{~kg})$ of $C$. japonica $\mathrm{D}$. Don. were exhaustively extracted with $\mathrm{Me}_{2} \mathrm{CO}$. The $\mathrm{Me}_{2} \mathrm{CO}$ extract was passed through a pad of charcoal, concd and re-extracted with EtOAc. The EtOAc-sol. portion ( $45 \mathrm{~g})$ was chromatographed on a silica gel column, eluting with gradients of hexane and EtOAc. Appropriate frs were comb. and purified by HPLC to give $18(13 \mathrm{mg}), 13(4 \mathrm{mg}), 15(9 \mathrm{mg})$, 16 (8 mg), 14 (110 mg), 3 (10 mg), 2 (55 mg), 4 (12 mg), 12 
<smiles>O=C1c2c(O)cc(O)cc2O[C@@H](c2ccc(O)c(O)c2)C1O</smiles>

1<smiles>[R]C1Cc2c(OC(C)=O)cc(OC(C)=O)cc2O[C@@H]1c1ccc(OC(C)=O)c(OC(C)=O)c1</smiles>

$6 \quad \mathrm{R}=\beta-\mathrm{OAc}$

$7 \mathrm{R}=\alpha-\mathrm{OAc}$<smiles>[R]Oc1cc(OC)cc(-c2oc3cc(OC)cc(O)c3c(=O)c2[R])c1</smiles><smiles>[R]Oc1cc(O)c2c(=O)c([R])c(-c3ccc([R])c([R])c3)oc2c1</smiles>

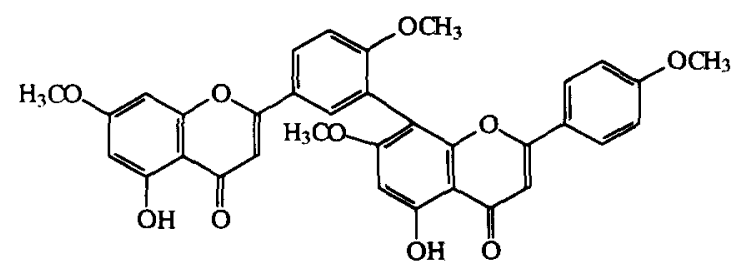

8<smiles>[R]C1(Cc2ccc(O)c(OC)c2)C(=O)OC[C@H]1Cc1ccc(O)c(OC)c1</smiles>

$9 R=H$ $10 \mathrm{R}=\mathrm{OH}$<smiles>COc1ccc([C@H]2c3cc(OC)c(OC)cc3CC(COC(C)=O)[C@H]2COC(C)=O)cc1OC</smiles>

11<smiles>COC1=CC(OC)C(OC)C(OC)C1</smiles>

12<smiles>[R]C1=CC(CCCO[Na])=CC2C1OC(c1ccc(OC(=O)OC)c(OC)c1)C2COC</smiles>

$13 \quad \mathrm{R}=\mathrm{H}$

$14 \quad \mathrm{R}=\mathrm{OCH}_{3}$

$15 R=O A C$<smiles>COC(C)=O</smiles>

16<smiles>COCCCC1C=C(OC)C(OC(C)=O)=C(C(COC)CC2CCC(OC(C)=O)C(OC)C2)C1</smiles>

17<smiles>COC(=O)C[C@H](CC=Cc1ccc(OC(C)=O)cc1)[C@H](C)C(=O)O[Na]</smiles>

18

(17 mg), 17 (20 mg), 6 (13 mg), 7 (15 mg), 11 (15 mg), 122.9 (C-5), 124.6 (C-6'), 126.0 (C-1'), 129.2 (C-2'), 134.0 $8(14 \mathrm{mg}), 9(15 \mathrm{mg}), 10(12 \mathrm{mg}), 5(10 \mathrm{mg})$ and $1(15 \mathrm{mg}), \quad\left(\mathrm{C}-3^{\prime}\right), 139.5(\mathrm{C}-4), 140.1(\mathrm{C}-1), 151.3(\mathrm{C}-3), 158.0\left(\mathrm{C}-4^{\prime}\right)$, in order of increasing polarity, respectively. $20.6,20.8,21.0,168.9,170.8,171.1(3 \times \mathrm{OAc})$.

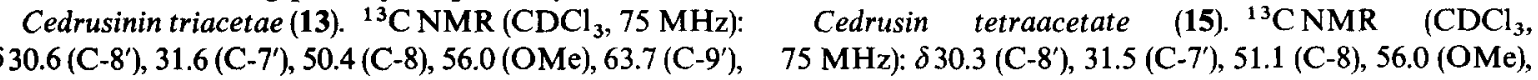
65.7 (C-9), 87.0 (C-7), 109.5 (C-5'), 109.7 (C-2), 118.0 (C-6), 63.7 (C-9'), 65.5 (C-9), 87.9 (C-7), 109.6 (C-2), 117.5 (C-6), 
Table 1. ${ }^{1} \mathrm{H}$ and ${ }^{13} \mathrm{C}$ NMR spectral data of compounds 16 and $17\left(\mathrm{CDCl}_{3}\right.$ solution, $\delta, J$ values in $\left.\mathrm{Hz}\right)$

\begin{tabular}{|c|c|c|c|c|}
\hline & \multicolumn{2}{|c|}{$\delta_{\mathrm{H}}(300 \mathrm{MHz})$} & \multicolumn{2}{|c|}{$\delta_{\mathrm{C}}(75 \mathrm{MHz})$} \\
\hline & 16 & 17 & 16 & 17 \\
\hline 1 & - & - & 139.5 & 138.0 \\
\hline 2 & $7.01(d, J 2)$ & $6.60(d, J 2)$ & 110.6 & 113.2 \\
\hline 3 & - & - & 151.1 & 150.6 \\
\hline 4 & - & - & 139.3 & 138.2 \\
\hline 5 & $7.0(d, J 8)$ & $6.85(d, J 8)$ & 122.6 & 122.4 \\
\hline 6 & $6.95(d d, J 2,8)$ & $6.64(d d, J 2,8)$ & 118.8 & 121.0 \\
\hline 7 & $5.82(d, J 7.5)$ & $\begin{array}{l}2.86(d d, J 7,11) \\
2.90(d d, J 7,11)\end{array}$ & 86.5 & 37.9 \\
\hline 8 & $3.80(d d d, J 3,6,7.5)$ & $3.44(d d d d, J 7,7,7,7)$ & 46.0 & 39.2 \\
\hline 9 & $\begin{array}{l}3.82(d d, J 6,11.5) \\
3.92(d d, J 3,11.5)\end{array}$ & $\begin{array}{l}4.16(d d, J 7,10.5) \\
4.22(d d, J 7,10.5)\end{array}$ & 63.6 & 66.4 \\
\hline $1^{\prime}$ & - & - & 128.6 & 139.6 \\
\hline $2^{\prime}$ & $6.66(d, J 2)$ & $6.54(d, J 2)$ & 117.1 & 119.2 \\
\hline $3^{\prime}$ & - & - & 135.4 & 134.1 \\
\hline $4^{\prime}$ & - & - & 146.2 & 136.5 \\
\hline $5^{\prime}$ & - & - & 144.2 & 151.0 \\
\hline $6^{\prime}$ & $6.64(d, J 2)$ & $6.62(d, J 2)$ & 113.0 & 110.7 \\
\hline $7^{\prime}$ & $2.62(t, J 7)$ & $2.59(t, J 7)$ & 32.0 & 32.3 \\
\hline $8^{\prime}$ & $1.92(t t, J 6.5,7)$ & $1.87(t t, J 6.5,7)$ & 30.6 & 30.2 \\
\hline $9^{\prime}$ & $4.08(t, J 6.5)$ & $4.05(t, J 6.5)$ & 63.7 & 63.7 \\
\hline \multirow[t]{2}{*}{$\mathrm{OMe}$} & $3.80(s)$ & $3.69(s)$ & 56.0 & 55.7 \\
\hline & $3.89(s)$ & $3.77(s)$ & 56.2 & 55.9 \\
\hline \multirow[t]{4}{*}{$\mathrm{OAc}$} & $1.81(s)$ & $1.94(s)$ & $20.5,168.8$ & $20.5,168.8$ \\
\hline & $2.05(s)$ & $2.05(s)$ & $20.6,170.5$ & $20.6,168.9$ \\
\hline & $2.28(s)$ & $2.25(s)$ & $21.0,171.1$ & $20.8,169.1$ \\
\hline & & $2.29(s)$ & & $20.9,171.0$ \\
\hline
\end{tabular}

Assignments of ${ }^{1} \mathrm{H}$ and ${ }^{13} \mathrm{C}$ resonances confirmed by $\mathrm{H}-\mathrm{C}$ COSY and $\mathrm{HMBC}$ spectra, as well as by NOE experiments.

$122.1\left(\mathrm{C}^{\prime} 6^{\prime}\right), 122.4\left(\mathrm{C}-2^{\prime}\right), 122.8(\mathrm{C}-5), 128.0\left(\mathrm{C}-1^{\prime}\right), 133.8$ $\left(\mathrm{C}-5^{\prime}\right), 135.0\left(\mathrm{C}-3^{\prime}\right), 139.5(\mathrm{C}-4), 139.8(\mathrm{C}-1), 149.0\left(\mathrm{C}-4^{\prime}\right)$, 151.4 (C-3), 20.2, 20.6, 20.8, 20.9, 168.9, 170.6, 170.7, 171.1 $(4 \times$ OAc).

Cis-Dihydrodehydrodiconiferyl alcohol triaacetate (16). Gum. $[\alpha]_{\mathrm{D}}^{25}-66^{\circ}\left(\mathrm{CHCl}_{3} ; c\right.$ 0.8). TLC (EtOAc$\mathrm{CH}_{2} \mathrm{Cl}_{2}, 1: 9$ ), $R_{f} 0.48$. IR $v_{\max }^{\text {neat }} \mathrm{cm}^{-1}: 1757,1728,1601$, 1494. UV $\lambda_{\max }^{\mathrm{MeOH}} \mathrm{nm}(\varepsilon): 281(5550), 255(1700), 211$ (40250). CD (MeOH): $[\theta]_{318}+670,[\theta]_{276.5}-2240$, $[\theta]_{253}+2700$. EIMS $(70 \mathrm{eV}) \mathrm{m} / \mathrm{z}$ (rel. int.) $486[\mathrm{M}]^{+}(4)$, $426(5), 384$ (25), 369 (5), 265 (6), $165(10), 43$ (100). HRMS for $\mathrm{C}_{26} \mathrm{H}_{30} \mathrm{O}_{9}$, requires 486.1890 ; found $[\mathrm{M}]^{+} \mathrm{m} / \mathrm{z}$ 486.1892 .

secoDihydrodehydrodiconiferyl alcohol tetraacetate (17). Gum. $[\alpha]_{\mathrm{D}}^{25}-2.5^{\circ}\left(\mathrm{CHCl}_{3} ; c 2.0\right)$. TLC (EtOAc$\mathrm{CHCl}_{3}$-hexane, $\left.1: 1: 1\right) \boldsymbol{R}_{f} 0.75$. IR $v_{\max }^{\text {neat }} \mathrm{cm}^{-1}: 1756,1730$, 1593, 1505. UV $\lambda_{\max }^{\mathrm{MeOH}} \mathrm{nm}(\varepsilon): 274$ (7700). CD (MeOH): $[\theta]_{264.5}+670,[\theta]_{286.5}-550$. EIMS $(70 \mathrm{eV}) \mathrm{m} / \mathrm{z}$ (rel. int.) $530[\mathrm{M}]^{+}(12), 488(75), 446(25), 428(60), 386(42)$, 189 (65), $137(90), 43(100)$. HRMS for $\mathrm{C}_{28} \mathrm{H}_{34} \mathrm{O}_{10}$, requires 530.2152; found $[\mathrm{M}]^{+} \mathrm{m} / z$ 530.2153.

Correlation of 17 with 14. A mixt. of $14(20 \mathrm{mg})$ and $10 \% \mathrm{Pd} / \mathrm{C}(5 \mathrm{mg})$ in $\mathrm{MeOH}(5 \mathrm{ml})$ was stirred at $20^{\circ}$ under an $\mathrm{H}_{2}$ atmosphere for $16 \mathrm{hr}$. The mixt. was filtered, the filtrate concd and purified by HPLC and elution with
EtOAc-hexane (4:1) to give secolignan 17a (18 mg). Gum. $[\alpha]_{\mathrm{D}}^{25}+39^{\circ}\left(\mathrm{CHCl}_{3} ; c 1.5\right)$. TLC (EtOAc-hexane, 4:1) $R_{f} 0.33$. IR $v_{\max }^{\text {neat }} \mathrm{cm}^{-1}: 3375,1597,1507,1487$, ${ }^{1} \mathrm{H} \mathrm{NMR}\left(\mathrm{CDCl}_{3}, 300 \mathrm{MHz}\right): \delta 1.78(t t, J=6.5,7 \mathrm{~Hz}$, H-8'), $2.57\left(t, J=7 \mathrm{~Hz}, \mathrm{H}-7^{\prime}\right), 2.85(d d, J=7,11 \mathrm{~Hz}, \mathrm{H}-7)$, $2.96(d d, J=7,11 \mathrm{~Hz}, \mathrm{H}-7), 3.42(d d d d, J=7,7,7,7 \mathrm{~Hz}$ $\mathrm{H}-8), 3.57\left(t, J=6.5 \mathrm{~Hz}, \mathrm{H}-9^{\prime}\right), 3.75(d d, J=7,11 \mathrm{~Hz}$, $\mathrm{H}-9), 3.76(s, \mathrm{OMe}), 3.83(s, \mathrm{OMe}), 3.85(d d, J=7,11 \mathrm{~Hz}$, $\mathrm{H}-9), 6.51\left(d, J=2 \mathrm{~Hz}, \mathrm{H}-2^{\prime}\right), 6.56\left(d, J=2 \mathrm{~Hz}, \mathrm{H}-6^{\prime}\right)$, $6.60(d, J=2 \mathrm{~Hz}, \mathrm{H}-2), 6.61(d d, J=2,8 \mathrm{~Hz}, \mathrm{H}-6), 6.73(d$, $J=8 \mathrm{~Hz}, \mathrm{H}-5) .{ }^{13} \mathrm{CNMR}\left(\mathrm{CDCl}_{3}, 75 \mathrm{MHz}\right): \delta 31.9(\mathrm{C}-$ $8^{\prime}$ ), 34.4 (C-7'), 36.6 (C-7), 44.3 (C-8), 55.8 (OMe), 55.9 (OMe), 62.1 (C-9'), 65.2 (C-9), 109.2 (C-6'), 111.7 (C-2), 114.0 (C-2'), 120.7 (C-6), 121.7 (C-5), 127.3 (C-3'), 132.3 (C-1), 133.0 (C-1'), 141.8 (C-4'), 143.6 (C-4), 146.2 (C-3),

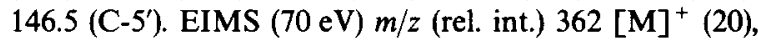
224 (5), 208 (67), 179 (25), 164 (15), 151 (12), 137 (100). HRMS for $\mathrm{C}_{20} \mathrm{H}_{26} \mathrm{O}_{6}$ requires 362.1730 ; found [M] ${ }^{+}$ $m / z 362.1731$.

Treatment of $17 \mathrm{a}(18 \mathrm{mg})$ with $\mathrm{Ac}_{2} \mathrm{O}(0.5 \mathrm{ml})$ in pyridine $(0.5 \mathrm{ml})$ for $16 \mathrm{hr}$ gave the corresponding tetraacetate $17(19 \mathrm{mg})$ after usual work-up.

Agatharesinol tetraacetate (18). Gum. $[\alpha]_{\mathrm{D}}^{25}-20^{\circ}$ $\left(\mathrm{CHCl}_{3} ; c \text { 1.3) [lit. [17] [ } \alpha\right]_{\mathrm{D}}^{25}-19^{\circ}\left(\mathrm{Me}_{2} \mathrm{CO} ; c\right.$ 1.0)]. ${ }^{13} \mathrm{CNMR}\left(\mathrm{CDCl}_{3}, 75 \mathrm{MHz}\right.$ ): $\delta 50.0$ (C-7), 64.0 (C-9), 72.8 
<smiles>COCC1c2cc(CCCOC(C)=O)cc(OC)c2OC1c1ccc(OC)c(OC)c1</smiles>

14

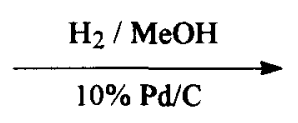

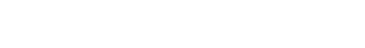<smiles>COc1cc(CC(CO)c2cc(CCCO)cc(OC)c2O)ccc1O</smiles>

17a

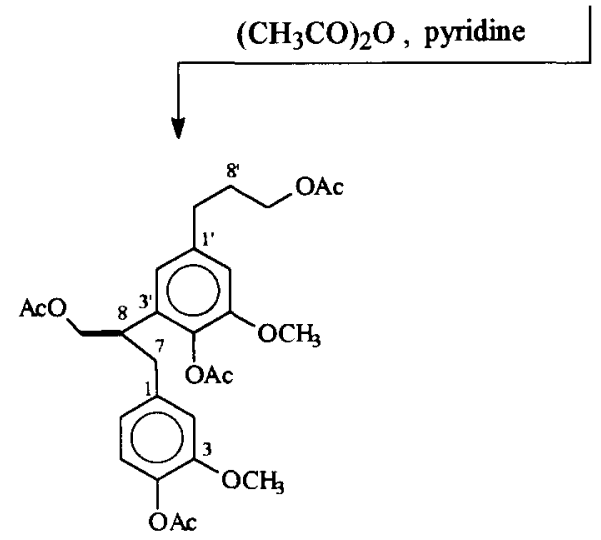

17

Scheme 1.

(C-8), $121.7\left(\mathrm{C}-3,5,3^{\prime}, 5^{\prime}\right), 127.3\left(\mathrm{C}-2^{\prime}, 6^{\prime}\right), 128.0\left(\mathrm{C}-8^{\prime}\right)$, $129.2(\mathrm{C}-2,6), 131.9\left(\mathrm{C}^{-} \mathbf{7}^{\prime}\right), 134.4\left(\mathrm{C}-1^{\prime}\right), 137.0(\mathrm{C}-1), 149.6$ (C-4), 150.2 (C-4'), 20.7, 20.7, 21.1, 21.1, 169.3, 169.4, 170.2, $170.6(4 \times \mathrm{OAc})$.

\section{REFERENCES}

1. Su, W.-C., Fang, J.-M. and Cheng, Y.-S. (1994) Phytochemistry 39, 603.

2. Su, W.-C., Fang, J.-M. and Cheng, Y.-S. (1994) Phytochemistry 35, 1279.

3. Su, W.-C., Fang, J.-M. and Cheng, Y.-S. (1994) Phytochemistry 37, 1109.

4. Su, W.-C., Fang, J.-M. and Cheng, Y.-S. (1993) Phytochemistry 34, 779.

5. Pew, J. C. (1948) J. Am. Chem. Soc. 70, 3031.

6. Chakravarti, D. and Ghosh, R. P. (1944) J. Indian Chem. Soc. 21, 171.

7. Guider, J. M., Simpson, T. H. and Thomas, D. B. (1955) J. Chem. Soc. 170.
8. Gomm, A. S. and Nierenstein, M. (1931) J. Am. Chem. Soc. 53, 4408.

9. Ternai, B. and Markham, K. R. (1976) Tetrahedron 32, 565:

10. Hergert, H. L. and Kurth, E. F. (1953) J. Org. Chem. 18, 521.

11. Okuyama, T., Ohta, Y. and Shibata, S. (1979) Shoyakugaku Zasshi 33, 185.

12. Haworth, R. D. and Richardson, T. (1935) J. Chem. Soc. 633.

13. Nishibe, S., Hisada, S. and Inagaki, I. (1971) Phytochemistry 10, 2231.

14. Fonseca, S. F., Campello, J. P., Barata, L. E. S. and Ruveda, E. A. (1978) Phytochemistry 17, 499.

15. Agrawal, P. K. and Rastogi, R. P. (1982) Phytochemistry 21, 1459.

16. Agrawal, P. K., Agrawal, S. K. and Rastogi, R. P. (1980) Phytochemistry 19, 1260.

17. Enzell, C. R. and Thomas, B. R. (1965) Acta Chem. Scand. 19, 913. 